\title{
SPECIFIC IN VIVO LABELING WITH GFP RETROVIRUSES, LENTIVIRUSES, AND ADENOVIRUSES FOR IMAGING
}

\author{
Robert M. Hoffman $^{1,2}$, Hiroyuki Kishimoto ${ }^{1,2,3}$, and Toshiyoshi Fujiwara ${ }^{3}$ \\ 1 AntiCancer, Inc., 7917 Ostrow Street, San Diego, CA 92111-3604 \\ 2 Dept. of Surgery, University of California, 200 West Arbor Dr., San Diego, CA 92103-8220 \\ 3 Div. of Surgical Oncology, Dept. of Surgery, Okayama University Graduate School of Medicine, \\ Dentistry and Pharmaceutical Sciences, 2-5-1 Shikata-cho, Okayama 700-8558, Japan
}

\begin{abstract}
Fluorescent proteins have revolutionized the field of imaging. Our laboratory pioneered in vivo imaging with fluorescent proteins. Fluorescent proteins have enabled imaging at the subcellular level in mice. We review here the use of different vectors carrying fluorescent proteins to selectively label normal and tumor tissue in vivo. We show that a GFP retrovirus and telomerase-driven GFP adenovirus can selectively label tumors in mice. We also show that a GFP lentivirus can selectively label the liver in mice. The practical application of these results are discussed.
\end{abstract}

Keywords: green fluorescent protein, mouse models, cancer, liver, selective labeling

\section{INTRODUCTION}

Fluorescent proteins can be used to visualize primary tumor growth, tumor cell motility and invasion, metastatic seeding and colonization, angiogenesis, and the interaction between the tumor and its microenvironment $(1,2)$. Fluorescent proteins of many different colors have now been characterized, and these can be used to color-code cancer cells of a specific genotype or phenotype. For example, the behavior of highly metastatic cancer cells labeled with green fluorescent protein (GFP) and low metastatic cancer cells labeled with red fluorescent protein (RFP) can be directly compared in vivo (3). Alternatively, the host and the tumor can be differentially labeled with fluorescent proteins. For example, a transgenic mouse expressing GFP in all of its cells, or a transgenic mouse labeled with GFP in specific cells such as endothelial cells, transplanted with tumor cells expressing RFP, enable the interaction between the tumor cells and the host cells to be visualized in real time (4). Real-time tracking of tumor growth and metastasis can be carried out in the intact animal $(1,2)$. For single-cell resolution, reversible acute skin flaps have been used over many parts of the body (skin, brain, lung, liver, etc.) (5). Real-time imaging with fluorescent proteins is especially important when evaluating the efficacy of therapeutics on metastasis and tumor recurrence (6).

Fluorescent proteins have very high extinction coefficients ranging from 6,500 up to 95,000 (7). In addition, they have very high quantum yields ranging from 0.24 up to $0.8(8)$. These properties make fluorescent proteins very bright. The large two-photon absorption of GFP is important for in vivo applications. Another important feature is the spectral distinction between many members of the family of fluorescent proteins. Therefore, a set of multicolor fluorescent proteins can be used simultaneously for multifunctional in vivo imaging. These properties make fluorescent proteins optimal for cellular imaging in vivo (1).

Our laboratory pioneered the use of GFP for in vivo imaging in 1997 (9). With the use of GFP, individual cancer cells could be observed for the first time in fresh unstained tissue or even the live animal. In 2000, we showed that GFP could be used for whole-body imaging (2).

To noninvasively image at the cellular level in live animals in real time, we developed a new imageable three-color animal model. The model consists of GFP-expressing mice transplanted with dual-color cancer cells labeled with GFP

Small Animal Whole-Body Optical Imaging Based on Genetically Engineered Probes edited by Alexander P. Savitsky, Robert E. Campbell, Robert M. Hoffman

Proc. of SPIE Vol. 6868, 68680F, (2008) - 1605-7422/08/\$18 · doi: 10.1117/12.773308

Proc. of SPIE Vol. 6868 68680F-1 
in the nucleus and red fluorescent protein in the cytoplasm. The Olympus IV100 Laser Scanning Microscope, with ultra-narrow microscope objectives ("stick objectives"), was used for three-color whole-body imaging of the two-color cancer cells interacting with the GFP-expressing stromal cells. Various in vivo phenomena of tumor-host interaction and cellular dynamics were imaged, including mitotic and apoptotic tumor cells, stromal cells interacting with the tumor cells, tumor vasculature, and tumor blood flow. This new model system enables the first cellular and subcellular images of unperturbed tumors in the live intact animal. New visible real-time targets for novel anticancer agents are provided in this model, including the color-coded interacting cancer and stromal cells, tumor vasculature, and blood flow (10).

\section{MATERIALS AND METHODS}

\subsection{In Vivo Labeling Of Tumors With GFP Retrovirus}

2.1.1 GFP retroviral vector. pLEIN, a retroviral vector, was purchased from Clontech (Palo Alto, Calif). pLEIN expresses enhanced GFP and the neomycin resistance gene on the same bicistronic message (11).

2.1.2 Cell culture. PT67, an NIH 3T3-based packaging cell line expressing the 10A1 viral envelope, was purchased from Clontech. PT67 cells were cultured in Dulbecco's modified Eagle's medium (Irvine Scientific, Santa Ana, Calif) supplemented with 10\% heat-inactivated fetal bovine sera (Gemini Bio-Products, Calabasas, Calif), $100 \mathrm{U} / \mathrm{mL}$ penicillin, and $100 \mu \mathrm{g} / \mathrm{mL}$ streptomycin.23 NUGC-4 cells were a kind gift of Dr. Narita (Laboratory of Experimental Pathology, Aichi Cancer Center Research Institute, Nagoya, Japan).28 NUGC-4 cells were cultured in RPMI 1640 (Life Technologies, Grand Island, NY) supplemented with $10 \%$ heat-inactivated fetal bovine sera, $100 \mathrm{U} / \mathrm{mL}$ penicillin, and $100 \mathrm{mg} / \mathrm{mL}$ streptomycin (11).

2.1.3 Retroviral transduction of packaging cells. PT67 packaging cells were plated at a density of $1 \times 10^{5}$ cells in a 6well plate 24 hours before transfection. The cells were $70-80 \%$ confluent at the time of GFP retrovirus transduction. A total of $2.5 \mu \mathrm{g}$ of plasmid and $15 \mu \mathrm{L}$ of $N$-(1-[2,3-dioleoyloxy] propyl)- $N, N, N$-trimethylammonium methylsulfate reagent Boehringer Mannheim, Indianapolis, Ind) were mixed according to the manufacturer's protocol. The cells were examined by fluorescence microscopy 48 hours after transfection. For selection, the cells were cultured in the presence of $0.5-2.0 \mathrm{mg} / \mathrm{mL}$ G418 (Life Technologies) for 14 days (11).

2.1.4 Preparation of retroviral supernatant. Retroviral GFP producer packaging cells were cultured at $80 \%$ confluence for 24 hours to generate retroviral supernatants. Supernatants were harvested, passed through a 0.45 -mm filter, and stored at $-80^{\circ} \mathrm{C}(11)$.

2.1.5 Viral titer determination. NIH 3T3 cells were plated at a density of $1 \times 10^{5}$ cells in 10 -cm dishes 24 hours before infection. The cells, at $70 \%$ confluenceat the time of infection, were incubated in $5 \mathrm{~mL}$ of undiluted and diluted retroviral supernatants. Selection in G418 $(1.0 \mathrm{mg} / \mathrm{mL})$ began at 48 hours postinfection. After 14 days, cells were stained with $0.5 \%$ methylene blue dissolved in 50\% methanol; G418-resistant colonies were counted (11).

2.1.6 Tumor transplantation. A total of $1 \times 10^{7}$ NUGC-4 cells in $1 \mathrm{~mL}$ of RPMI 1640 were inoculated subcutaneously into 6- to 8-week-old BALB/c $n u / n u$ female mice. At 5 weeks postinoculation, the subcutaneous tumor was excised and cut into pieces that could pass through an 18-gauge needle. Tumor pieces were suspended in RPMI 1640, and $1 \mathrm{~mL}$ of suspension was injected with an 18-gauge needle into the peritoneal cavity. All mice were engrafted at the same time (11).

2.1.7 In vivo transduction of GFP. A total of $1 \mathrm{~mL}$ of the GFP retroviral supernatant, produced as described above, was supplemented with $8.0 \mu \mathrm{g} / \mathrm{mL}$ polybrene and was administrated to the tumor-bearing mice once per day intraperitoneally (i.p.) from day 4 to day 10 after tumor transplantation (11).

2.1.8 Visualization of tumor growth, spread, and metastasis by GFP fluorescence. Mice were anesthetized by isoflurane inhalation and put in a supine position. A laparotomy was performed via a midline incision. Fresh visceral organs were analyzed under a fluorescence microscope with GFP filters (Chroma Technology Corp., Brattleboro, Vt). After observation, the abdominal wall and the skin were closed with 6-0 silk sutures (11). 


\subsection{In Vivo Labeling of Liver with GFP Lentivirus}

2.2.1 Animals. Twelve-week-old female nude (Hsd:Athymic Nude- $n u$ ) mice (Harlan Sprague-Dawley, Indianapolis, IN) were used (12).

2.2.2 Virus production. A third-generation, Tat-free packaging system was used to produce recombinant lentivirus. The GFP-LV plasmid together with the two packaging plasmids [encoding human immunodeficiency virus (HIV) gag, pol, and rev] and the plasmid coding for VSV-G envelope were transfected into 293T(HEK) cells using the calcium phosphate method. In brief, we transfected $2415-\mathrm{cm}$ dishes and harvested the virus by collecting the cell culture medium 24, 48, and $72 \mathrm{~h}$. After filtering the collected medium through $0.45-\mathrm{mm}$ filters, the virus was concentrated by spinning at $68,400 \mathrm{~g}$ for $2 \mathrm{~h}$ followed by a second spin $(59,000 \mathrm{~g}, 2.5 \mathrm{~h}$ at room temperature). The resulting pellet was resuspended in $200 \mathrm{ml}$ Hanks' buffer. The titer of lentiviral vectors was determined by measuring the amount of HIV p24 Gag antigen by ELISA (Alliance; NEN Life Science Products, Boston, MA) (12).

2.2.3 In vivo fluorescence optical imaging. A Leica stereomicroscope (Model LZ12) equipped with a mercury 50-W lamp was used with a Hamamatsu C5810 three-chip cooled color charge-coupled-device camera (Hamamatsu Photonics Systems, Hamamatsu City, Japan) attached. Excitation of GFP was achieved at $470 \mathrm{~nm}$ (using a D425/60 band-pass filter and 470 DCXR dichroic mirror). The emitted fluorescence was collected through a long-pass filter (GG475; Chroma Technology, Brattleboro, VT). Images were analyzed using Image Pro Plus 3.1 software (Media Cybernetics, Silver Springs, MD). Images were captured directly on an IBM PC or recorded on a high-resolution Sony VCR, Model SLV-R1000 (Sony, Tokyo) (12).

\subsection{For In Vivo Labeling of Metastatic Cancer with a Telomerase-Driven Adenovirus}

2.3.1 OBP-401. OBP-401 is a telomerase-specific replication-competent adenovirus variant in which the hTERT promoter element drives the expression of $E 1 A$ and $E 1 B$ genes linked with an internal ribosome entry site, with the GFP gene inserted under the CMV promoter into the E3 region for monitoring viral replication. The virus was purified by ultracentrifugation in cesium chloride step gradients, their titers were determined by a plaque-forming assay using 293 cells and they were stored at $-80^{\circ} \mathrm{C}(13)$.

2.3.2 Subcutaneous tumor model. Tumor cells were trypsinized, washed three times with PBS, and resuspended in Hank's balanced salt solution (HBSS). Three $\times 10^{6}$ PC-3-RFP cells were subcutaneously injected with a 27 -gauge needle. When tumors reached $6 \sim 7 \mathrm{~mm}$ in diameter, OBP-401 $\left(10^{8} \mathrm{PFU} / 0.1 \mathrm{ml}\right.$ of PBS $)$ was injected i.t. Mice were anaesthetized with ether, and examined for GFP expression.

2.3.3 Peritoneal dissemination model. HCT116-RFP cells ( 3 X $\left.10^{6}\right)$ were suspended in $0.2 \mathrm{ml}$ of HBSS, and inoculated into the abdominal cavity of $n u / n u$ mice with a 27 -gauge needle. 12 days after tumor cell inoculation, OBP-401 (10 PFU/0.2 ml of PBS) was injected into the abdominal cavity with the same technique. Five days after virus injection, the animals were sacrificed under ether anesthesia and examined via a laparotomy. The abdominal cavity was analyzed under fluorescence imaging.

2.3.4 Liver metastastic model. HCT116 cells $\left(2 \times 10^{6}\right)$ were suspended in $0.05 \mathrm{ml}$ Matrigel, and injected into the spleen of $n u / n u$ mice with a 28 -gauge needle. 10 days after tumor cell inoculation, OBP-401 (10 8 PFU/0.1 ml of PBS) was injected into the tail vein. Five days after virus injection, mice were examined via a laparotomy. The liver was analyzed by fluorescence imaging. One week after the first open examination, mice had a second look observation.

\section{RESULTS}

\subsection{Retrovirus to label tumors in live mice with GFP}

In order to translate GFP imaging technology to the clinic, we are developing vectors to deliver GFP to the living animal to specifically label tumors or other tissues. The GFP gene on a retroviral vector was administered to intraperitoneally (i.p.) growing human stomach cancer in nude mice to visualize future regional and distant metastases. GFP retroviral supernatants were injected i.p. from day 4 to day 10 after i.p. implantation of the cancer cells. Tumor and metastasis fluorescence was visualized every other week with the use of fluorescence optics via a laparotomy on the 
tumor-bearing animals. At 2 weeks after retroviral GFP delivery, GFP-expressing tumor cells were observed in gonadal fat, greater omentum, and intestine, indicating that these primary i.p.-growing tumors were efficiently transduced by the GFP gene and could be visualized by its expression. At the second and third laparotomies, GFP-expressing tumor cells were observed to have spread to lymph nodes in the mesentery and other regional sites. At the fourth laparotomy, widespread tumor growth was visualized by GFP expression, inducing liver metastasis. No normal tissues were found to be transduced by the GFP retrovirus. Thus, reporter gene transduction of the primary tumor enabled detection of its subsequent metastasis. This gene therapy model could be applied to primary tumors before resection or other treatment in order to have a fluorescent early detection system for metastasis and recurrence (11).

\subsection{Lentivirus to label the liver with GFP in mice}

Lentiviral vectors are a promising tool for gene therapy. They have been shown to transduce a broad spectrum of nondividing cells in vivo, such as neurons, retina, liver, muscle, and hematopoietic stem cells. The ability to infect nondividing liver cells is highly desirable feature of gene therapy vectors since most of the liver cells normally do not divide (12).

An HIV-1-based lentiviral vectors that contain the GFP coding sequence was used for in vivo labeling of the liver (GFPLV). To enhance transgene expression and transduction of the target cells, the woodchuck hepatitis virus posttranscriptional element (WPRE) and a central polypurine tract were included in the vectors. Transduction of the liver by GFP-LV was monitored in the living animal using in vivo fluorescence optical imaging (12).

Six days after injection of GFP-LV (IU) into the portal vein of nude mice, green fluorescence was detectable in the liver of the injected animals using in vivo imaging. At day 21 all liver lobes of mice injected with GFP-LV exhibited a homogeneous green fluorescence, but not in the PBS-injected control. Intraperitoneal (ip) injection of GFP-LV also resulted in a high level of transduction of the liver and the spleen, while in the PBS-injected controls no fluorescence was detectable. GFP expression was detectable even 6 months after injection. Western blot analysis demonstrated a dose-dependent effect of the lentiviral vector on liver GFP expression. In addition, inclusion of a cppt element further increased (two- to three-fold) GFP expression. PCR analysis of proviral DNA showed vector integration in the liver 3 weeks after injection of the lentiviral vectors (12).

GFP expression in the liver was further analyzed by confocal microscopy of frozen sections 21 days after GFP-LV injection. Sections of transduced livers exhibited a strong green fluorescence throughout the whole organ. The majority of GFP-positive cells were nonhepatocytes, such as perisinusoidal cells, Kupffer cells, and endotheliocytes. However, hepatocytes present in the sections were also GFP positive. The GFP-positive hepatocytes were not labeled with BrdU indicating they were not dividing. The results showed that cell cycle progression is not required for transduction of hepatocytes in vivo. No significant changes in serum alanine aminotransferase (ALT) concentrations were observed after injection of lentiviral vectors into the portal vein (12).

\subsection{Telomerase-driven adenovirus to label tumors in mice}

We have developed a new approach for visualizing tumors whose fluorescence can be detected using telomerasespecific replication-competent adenovirus expressing GFP (OBP-401). OBP-401 contains the replication cassette, in which the human telomerase reverse transcriptase (hTERT) promoter drives expression of E1 genes, and the GFP gene for monitoring viral replication. When OBP-401 was intratumorally injected into HT29 tumors orthotopically implanted into the rectum in BALB/c $n u / n u$ mice, para-aortic lymph node metastasis could be visualized at laparotomy under a three-chip color cooled charged-coupled device camera. These results indicate that OBP-401 causes viral spread into the regional lymphatic area and selectively replicates in neoplastic lesions, resulting in GFP expression in metastatic lymph nodes. This technology is adaptable to detect lymph node metastasis in vivo as a preclinical model of surgical navigation (13).

After intratumoral injection of OBP-401, whole-body images of mice with subcutaneous SW620 and HT29 tumors showed that intratumoral GFP fluorescence was detectable within 24 hours after local delivery of viruses. The fluorescence intensity reached maximum levels within 4 days after injection and was maintained for at least 7 days. High GFP expression was visible on the surface of tumors as well as across serially sliced sections. No GFP fluorescence was detected when non-tumor-bearing mice were subcutaneously injected with of OBP-401 (13). 
GFP expression was detected from $24 \mathrm{~h}$ after intratumoral administration of OBP-401 in primary orthotopicallyimplanted rectal tumors, with maximum signal occurring 2-4 d after injection. After injections of OBP-401 into the orthotopically-implanted rectal tumors, GFP was detected in 12 of 13 metastatic lymph nodes. The results indicate that intratumoral injection of OBP-401 causes viral spread into the regional lymphatic area and selective replication in cancer cells in metastatic lymph nodes, which in turn could be imaged with GFP fluorescence (13).

With an intraperitoneal dissemination model of human colon cancer HCT-116 in nude mice, we were able to visualize almost all the peritoneal disseminated nodules by GFP expression five days after injection of OBP-401 into the abdominal cavity.

We have selectively labeled liver metastasis with OBP-401. Human colon cancer HCT-116 cells were implanted intrasplenically with resultant liver metastases. The liver metastases were labeled with GFP after systemic administration of OBP-401. Five days after systemic administration of $10^{8} \mathrm{PFU}$ OBP-401, all the HCT-116 metastatic liver tumor nodules were visualized by GFP fluorescence.

We have also examined whether OBP-401 might be useful for labeling peritoneal dissemination of colon cancer in mice for the purpose of surgical navigation. Peritoneally disseminated nodules of human colon cancer HCT-116 in nude mice were visualized by GFP expression five days after ip injection of OBP-401 into the abdominal cavity. The peritoneal tumors were then resected under fluorescence guidance.

We have also developed a practical and simple manual means to image tumors expressing GFP in the living animal that could readily be applied to the operating theater. A blue LED flashlight (LDP LLC, Woodcliff Lake, NJ, USA; www.maxmax.com/OpticalProducts.htm) with an excitation filter (midpoint wavelength peak of $470 \mathrm{~nm}$ ) and a D470/40 emission filter (Chroma Technology, Brattleboro, VT, USA) could be used for whole-body imaging of mice with GFP and RFP-expressing tumors growing in or on internal organs. The images were readily seen by the naked eye with no anesthesia, substrate, or restraint of the animal needed. This simple flashlight filter method along with filter goggles will enable fluorescence guided surgery with the methods described (14). 


\section{REFERENCES}

1. R.M. Hoffman, "The multiple uses of fluorescent proteins to visualize cancer in vivo," Nat. Rev. Cancer 5, pp. 796-806, 2005.

2. M. Yang, E. Baranov, P. Jiang, F-X. Sun, X-M. Li, L. Li, S. Hasegawa, M. Bouvet, M. Al-Tuwaijri, T. Chishima, H. Shimada, A.R. Moossa, S. Penman, R.M. Hoffman, "Whole-body optical imaging of green fluorescent protein-expressing tumors and metastases”, Proc. Natl. Acad. Sci. USA 97, pp. 1206-1211, 2000

3. N. Yamamoto, M. Yang, P. Jiang, M. Xu, H. Tsuchiya, K. Tomita, A.R. Moossa, R.M. Hoffman, "Determination of clonality of metastasis by cell-specific color-coded fluorescent-protein imaging," Cancer Res. 63, pp. 7785-7790, 2003.

4. M. Yang, J. Reynoso, P. Jiang, L. Li, A.R. Moossa, R.M. Hoffman, "Transgenic nude mouse with ubiquitous green fluorescent protein expression as a host for human tumors," Cancer Res. 64, pp. 8651-8656, 2004.

5. M. Yang, E. Baranov, J.W. Wang, P. Jiang, X. Wang, F-X. Sun, M. Bouvet, A.R. Moossa, S. Penman, R.M. Hoffman, "Direct external imaging of nascent cancer, tumor progression, angiogenesis, and metastasis on internal organs in the fluorescent orthotopic model," Proc. Natl. Acad. Sci. USA 99, pp. 3824-3829, 2002.

6. M. Katz, M. Bouvet, S. Takimoto, S., D. Spivack, A.R. Moossa, R.M. Hoffman, "Survivalefficacy of adjuvant cytosine-analogue CS-682 in a fluorescent orthotopic model of human pancreatic cancer," Cancer Res. 64, pp. $1828-1833,2004$.

7. V. Verkhusha, K.A. Lukyanov, "The molecular properties and applications of Anthozoa fluorescent proteins and chromoproteins," Nat. Biotechnol. 22, pp. 289-296, 2004.

8. M. Zimmer, "Green fluorescent protein (GFP): applications, structure and related photophysical behavior," Chem. Rev. 102, pp. 759-781, 2002.

9. T. Chishima, Y. Miyagi, X. Wang, H. Yamaoka, H. Shimada, A.R. Moossa, R.M. Hoffman, “Cancer invasion and micrometastasis visualized in live tissue by greenfluorescent protein expression," Cancer Res. 57, pp. 2042-2047, 1997.

10. M. Yang, P. Jiang, R,M. Hoffman, "Whole-body subcellular multicolor imaging of tumor-host interaction and drug response in real time”, Cancer Res. 67, pp. 5195-5199, 2007

11. S. Hasegawa, M. Yang, T. Chishima, H. Shimada, A.R. Moossa, R.M. Hoffman, "In vivo tumor delivery of the green fluorescent protein gene to report future occurrence of metastasis," Cancer Gene Ther. 7, pp. 1336-1340, 2000 .

12. A. Pfeifer, T. Kessler, M. Yang, E. Baranov, N. Kootstra, D.A. Cheresh, R.M. Hoffman, I. Verma, "Transduction of liver cells by lentiviral vectors: Analysis in living animals," Mol. Ther. 3, pp.319-322, 2001.

13. H. Kishimoto, T. Kojima, Y. Watanabe, S. Kagawa, T. Fujiwara, F. Uno, F. Teraishi, S. Kyo, H. Mizuguchi, Y. Hashimoto, Y. Urata, N. Tanaka, T. Fujiwara, "In vivo imaging of lymph node metastasis with telomerasespecific replication-selective adenovirus," Nat. Med. 12, pp. 1213-1219, 2006.

14. M. Yang, G. Luiken, E. Baranov, R.M. Hoffman, "Facile whole-body imaging of internal fluorescent tumors in mice with an LED flashlight,” BioTechniques 39, pp. 170-172, 2005. 